Int. J. Dev. Biol. 49: 437-441 (2005)

doi: $10.1387 / \mathrm{ijdb} .051974 \mathrm{lb}$

Short Communication

\title{
Two members of the Fxr gene family, Fmr1 and Fxr1, are differentially expressed in Xenopus tropicalis
}

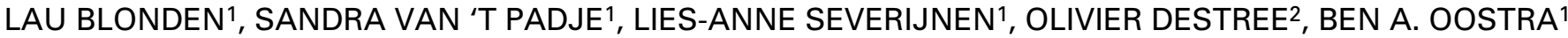 \\ and ROB WILLEMSEN*,1 \\ ${ }^{1}$ CBG Dept. of Clinical Genetics, Erasmus MC Rotterdam, The Netherlands and \\ ${ }^{2}$ Hubrecht Laboratory, Netherlands Institute for Developmental Biology, Utrecht, The Netherlands
}

\begin{abstract}
The Fxr gene family is composed of three members, FMR1, FXR1 and FXR2. The FMR1 gene is involved in the fragile $X$ syndrome, whereas for the other two members, no human disorder has been identified yet. An appropriate animal model to study in vivo gene function is essential to unravel the cellular function of the gene products FMRP, FXR1P and FXR2P, respectively. In Xenopus tropicalis both Fmr1 and Fxr1 were identified; however, unexpectedly Fxr2 was not. Here we describe the characterization of both Fmrp and Fxr1p in Xenopus tropicalis. Fmrp is expressed ubiquitously throughout the embryo during embryonic development, whereas Fxr1p shows a more tissue-specific expression particularly during late embryonic development. In adult frogs both proteins are highly expressed in most neurons of the central nervous system and in all spermatogenic cells in the testis. In addition, Fxr1p is also highly expressed in striated muscle tissue. Western blotting experiments revealed only one prominent isoform for both proteins using different tissue homogenates from adult frogs. Thus, for in vivo gene function studies, this relative simple animal model may serve as a highly advantageous and complementary model.
\end{abstract}

KEY WORDS: FMR1, FXR1, fragile X syndrome, costameres, mental retardation

FMRP, FXR1P and FXR2P belong to a small family of fragile Xrelated proteins ( $F X R$ family). Absence of FMRP in neurons of the central nervous system is the cause of the fragile $X$ syndrome, the most common inherited form of mental retardation in humans (Bardoni and Mandel, 2002). The fragile $X$ syndrome is a neurodevelopmental disorder characterized by immature dendritic spines and altered synaptic strength (Willemsen et al., 2004). The absence or dysfunction of both FXR1P and FXR2P has not yet been defined to a human disease; however, a function of FXR1P in striated muscle development has been suggested (Dube et al., 2000, Mientjes et al., 2004). The cellular function of FXR2P, like FMRP, seems to be related to learning processes (Bontekoe et al., 2002). For all three genes mouse models have been generated and specific phenotypes have been reported (Bakker et al., 1994, Bontekoe et al., 2002, Mientjes et al., 2004). Unfortunately, in vivo gene function studies in mammalians, including mice, focusing on the period of embryonic development are difficult. As a first step to generate a more suitable vertebrate animal model to study the physiological function of the three $F x r$ genes during embryonic development an initial characterization of the Fxr gene family has been performed in Xenopus tropicalis. Unlike the slow-growing, tetraploid Xenopus laevis, however,
Xenopus tropicalis is diploid and has a relatively short $(<5$ months) life cycle. A high level of sequence conservation of the FXR genes throughout various vertebrates (mouse, rat, zebrafish) has been demonstrated (Engels et al., 2004). This was used to screen the Sanger Institute $X$. tropicalis EST database with sequences from the human $F X R$ family members to find orthologues of the $X$. tropicalis $F X r$ genes. Interestingly, only complete coding sequences for both $X t F m r 1$ and $X t F X r 1$ could be compiled from overlapping ESTs. Using the JGI $X$. tropicalis genome assembly release v3.0 which includes a total of 33,749 gene models at a coverage of $7.4 \mathrm{X}$, combined with the Sanger Institute $X$. tropicalis EST database did not yield a potential $X$ tF $x$ r2 gene. Apparently, an ortholog of $F X R 2$ is absent in this particular species. Fig. 1A illustrates a comparison of human FMRP and FXR1P with the $X$. tropicalis Fxr-protein family. Overall identities between human and $X$. tropicalis FMRP and between human and $X$. tropicalis FXR1P was $76 \%$ and $82 \%$, respectively. Both $X$. tropicalis proteins contained the important functional domains,

Abbreviations used in this paper: FXR, fragile X-related; NES, nuclear export signal; NLS, nuclear localization signal.

\footnotetext{
*Address correspondence to: Dr. Rob Willemsen. Erasmus MC, Dept of Clinical Genetics, P.O. box 1738, 3000 DR Rotterdam, The Netherlands. Fax. +31-10-408-9461. e-mail: r.willemsen@erasmusmc.nl
} 
A
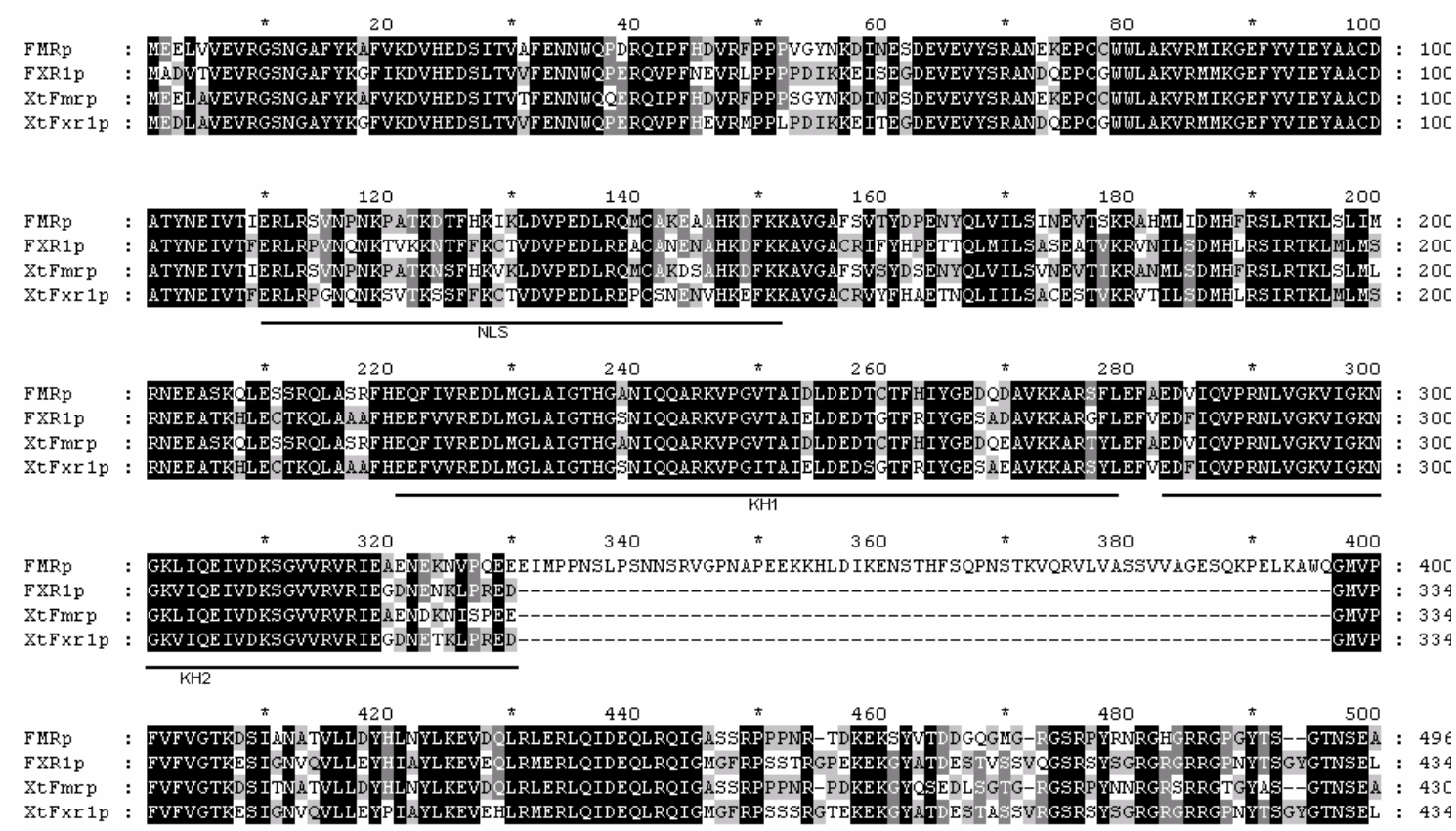

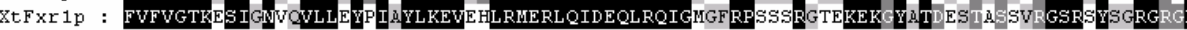
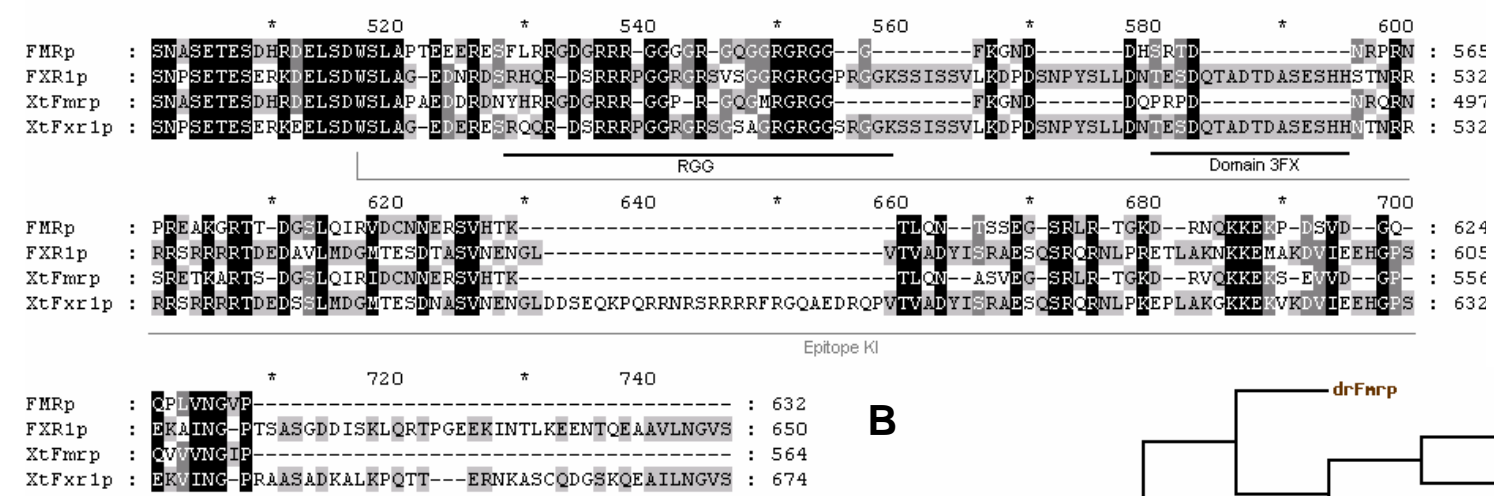

Fig. 1. Amino acid sequence alignment of $X$. tropicalis Fmrp and Fxr1p with their human orthologues. (A) Identical residues are shaded in black and conserved substitutions in grey. The alignment shows highly homologous regions of both Fmrp and Fxr1p between human and X. tropicalis. All orthologues contain two KH domains, a nuclear localisation signal (NLS), a nuclear export signal (NES) and an RGG box. In addition, the peptide sequence of the epitopes of the antibodies are depicted. (B) Phylogenetic analysis of the Fxr gene family members for human (FXR), mouse (Fxr), zebrafish (drFxr) and frog (XtFxr). The early evolutionary origin of this family is indicated by the Fmr1 like genes from Ciona, Hydractinia (hyFmr1) and fruitfly (dFmr1).

including the nuclear localisation signal (NLS), two KH domains, the nuclear export signal (NES) and an RGG box. The 63 amino acid region directly after the second $\mathrm{KH}$ domain that corresponds to exon 11 and 12 in human was missing from FMR1 in $X$. tropicalis, which is in accordance with earlier notions of $F M R 1$ obtaining these sequences during mammalian evolution. In contrast to mouse, both human and $X$. tropicalis show the presence of the largest transcript containing the 51 basepairs of exon 17.

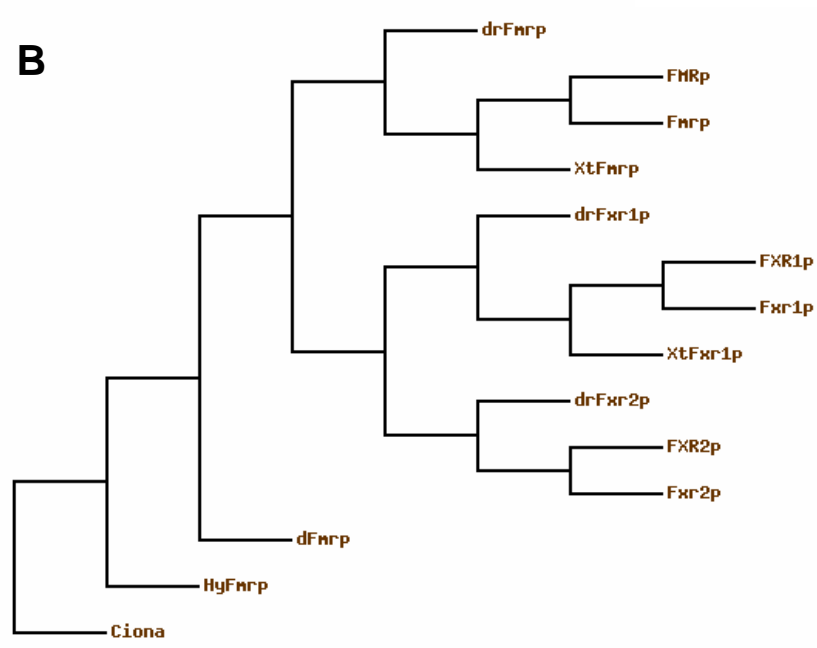

Interestingly, no CGG repeats were found in the 5' UTR sequences of the XtFmr1 gene. CGG repeat expansion to over 200 units is the mutational mechanism found in the vast majority of fragile $X$ patients. In addition, a phylogenetic analysis was performed, including human, mouse, zebrafish and frog proteins. A clear clustering of the respective $X$. tropicalis proteins with their predicted orthologues could be demonstrated (Fig. 1B). There is no indication for a separate development of the $X$. tropicalis Fxr 
family. The similar branch evolution of the three clusters makes an absence of $X t F \times r 2 p$ unexpected. Further Blast search through the JGI $X$. tropicalis genome database version 2.0 with the longest coding sequences for both $X$ tFmr1 and $X$ tF $X r 1$ revealed the genomic structure of both genes. Alignments of these virtual gene structures with known human/mouse data showed a $100 \%$ identical exonic structure (data not shown). Protein expression studies were performed with specific antibodies against both Fmrp and Fxr1p. First, transfection studies were performed to study the occurrence of cross-reactivity of our antibodies between the different Fxr-proteins. Both proteins share a high homology and knockout tissue from frogs is not available, thus, HEK293T cells were transfected with an expression construct containing either XtFmr1 or XtFXr1 preceded by an EGFP sequence. Expression of the construct was monitored by the presence of green fluorescent HEK293T cells. For the two fusionproteins (EGFP-Fmrp and EGFP-Fxr1p) no cross-reactivity with our anti- bodies could be observed (Fig. 2). Antibody KI specifically recognized Fmrp in HEK293T cells transfected with the EGFP-Fmr1 expression construct (Fig. 2B; red), whereas Fxr1p labelling was totally absent in HEK293T cells expressing EGFP-Fxr1p (Fig. 2D). Similar results were obtained with antibody 3FX against Fxr1p. Fig. 2F (red) illustrates the presence of Fxr1p with antibody 3FX using expression construct EGFP- $F \times r 1$ and the lack of crossreactivity with EGFP-Fmrp using construct EGFP-Fmr1(Fig. $2 \mathrm{H}$ ). Next, the cellular distribution of Fmrp was studied in embryonic stages and adult frogs using an indirect immunoperoxidase technique (Fig. 3). Paraffin sections of embryos at stages 6, 23, 37 and 46 of development representing $2 \mathrm{~h}, 1 \mathrm{~d}, 2 \mathrm{~d}$ and $7 \mathrm{~d}$ after fertilization, respectively, were studied. Fmrp showed a nuclear staining at 2 hours post fertilization (2 hpf; Fig. 3A) that changed to a cytoplasmic labelling throughout all cells in the later stages (Figs. 3 B-D). In adult frogs, Fmrp distribution showed a tissuespecific pattern. Fmrp was abundantly present in the cytoplasm of
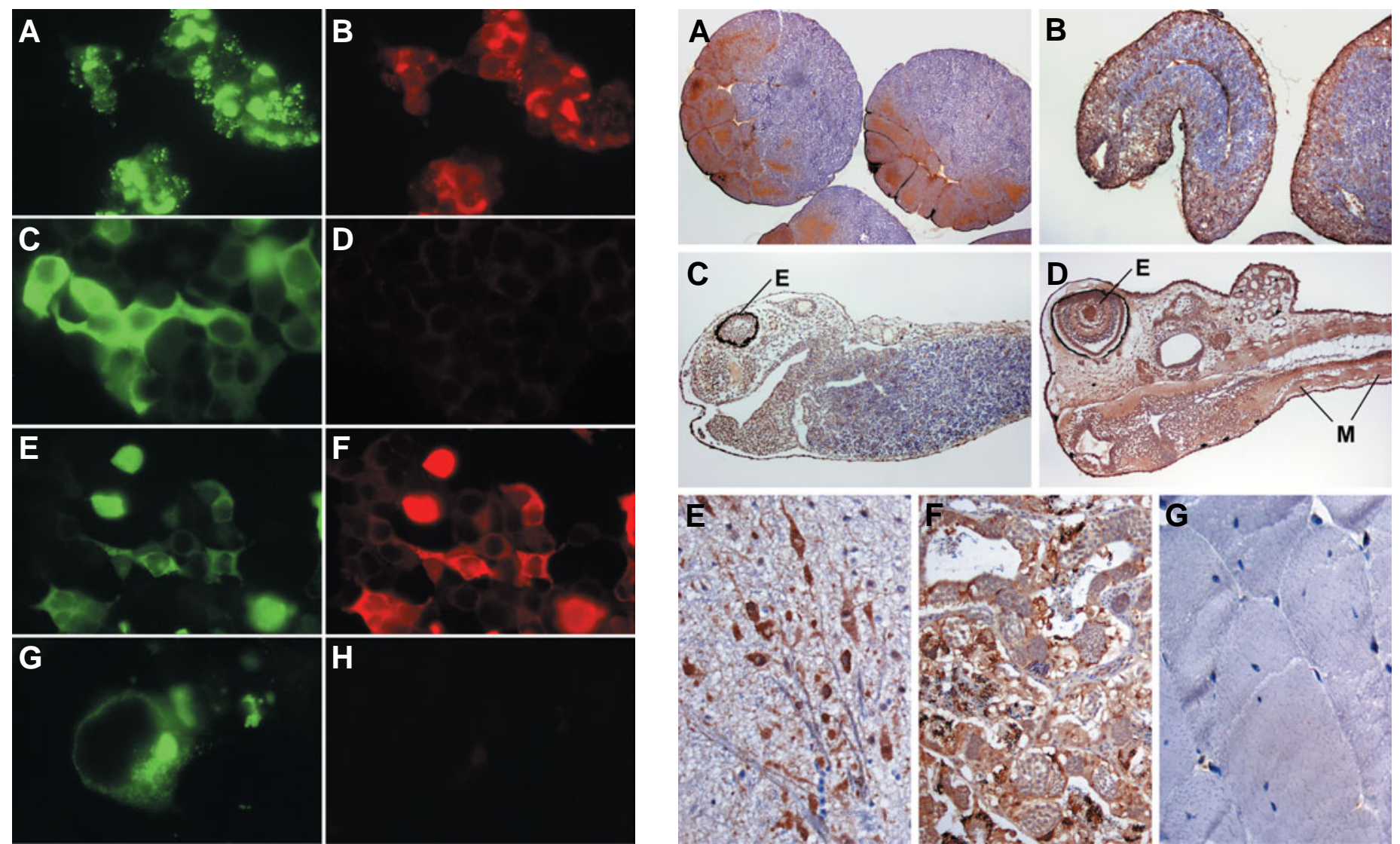

Fig. 2. (Left) Specificity of antibodies KI and 3FX against XtFmrp and XtFxr1p, respectively. GFP signal in HEK293T cells that were transfected with eitherXtFmr1-EGFP (A,G) orXtFxr1-EGFP (C,E). Cells were simultaneously stained immuno-cytochemically (red) using either antibody KI (B,D) or 3FX $(\mathbf{F}, \mathbf{H})$. Specific labelling of antibody KI was only observed in the XtFmr1 -EGFP transfected cells (B). The absence of KI positive-labelling in the XtFxr1 -EGFP transfected cells (D), indicates lack of cross-reaction of this antibody with Fxr1p. Similarly, antibody $3 F X$ shows only a specific labelling with Fxr1p (F) and no cross-reactivity with Fmrp (H).

Fig. 3. (Right) Fmrp distribution in $\boldsymbol{X}$. tropicalis embryos and adult frogs using antibody KI. Fmrp staining is present in the nuclei in stage 6 (A). The cells composing the animal pole show the typical large nucleus. At stage 23, when internally segregation of the brain occurs, Fmrp expression is evenly distributed in the cytoplasm of most cells (B). At stages 37 (C) and 46 (D), Fmrp expression remains ubiquitous with high expression in the eye, brain, intestine and skeletal muscle tissue. In contrast, adultX. tropicalis show a tissue-specifc expression pattern with high expression in the cytoplasm of most neurons in the brain (E) and all the spermatogenic cells of the testis (F). Note the absence of Fmrp in skeletal muscle (G). 
most neurons in the brain (Fig. 3E) and in all the spermatogenic cells of the testis (Fig. 3F). Notably, Fmrp could not be detected in striated muscle tissue (Fig. $3 \mathrm{G}$ for skeletal muscle). Our results for embryonic Fmrp expression in $X$. tropicalis are not completely in line with previous reports on mouse and zebrafish expression patterns that showed an overall expression during very early embryonic development that became tissue-specific at later stages (Bakker et al., 2000)(van't Padje, personal comm.). Apparently, the tissue-specific Fmrp expression in frogs occurs later in development compared to mouse and fish. Interestingly, the nuclear distribution of Fmrp during very early development ( $2 \mathrm{hpf}$ ) has been described for zebrafish as well (van't Padje, personal comm.). In adult frogs, a similar tissue-specific expression of Fmrp in brain and testis has been reported in human, mouse and fish. However, the presence of Fmrp in all spermatogenic cells of the testis has not been observed in the other vertebrates that showed only Fmrp expression in spermatogonia at the basal membrane of the seminiferous tubules (Bakker et al., 2000, Tamanini et al., 1997). In contrast, Fxr1p distribution shows a more tissue-specific pattern during embryonic development. At a very early stage (2 hpf), Fxr1p showed a nuclear staining like Fmrp (Fig. 4A). However, at stage 23 when internally segregation of the brain occurs a spatial organization of Fxr1p developed more preferentially in structures that will develop into muscle (mesoder-
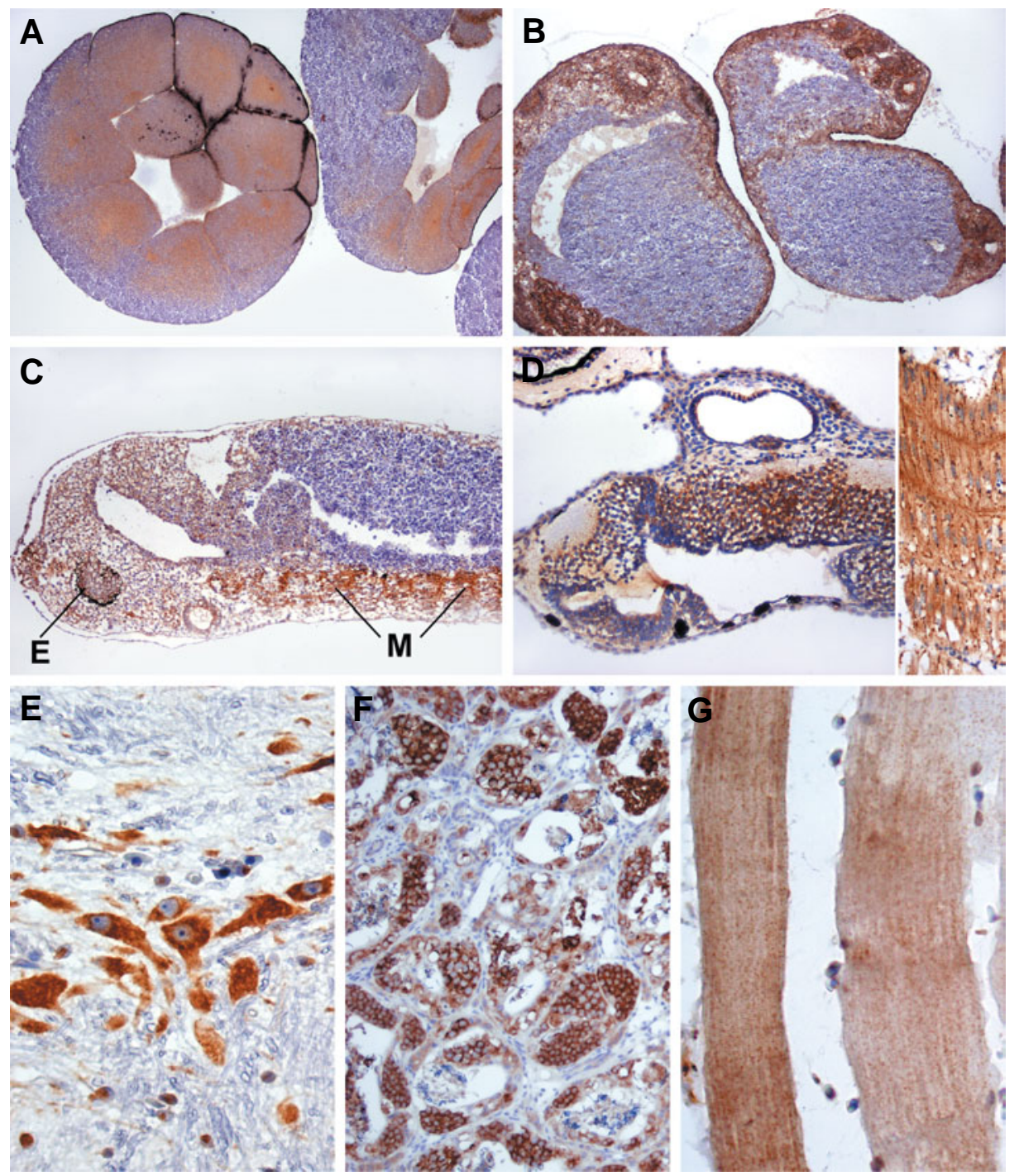

Fig. 4. Fxr1p distribution in $X$. tropicalis embryos and adult frogs using antibody 3FX. Fxr1p staining is detected in the nuclei of pre MBT cells (A) (=morula); stage 6. At stage 23, high Fxr1p expression is predominantly present in structures that will develop into muscle and brain tissue (B), although a weak labelling is also present in the other cell types. At stages 37 (C) and 46 (D), Fxr1p shows a tissue-specific expression pattern with a high expression in myoblasts of muscle tissue and neurons of the brain. The inset in (D) shows a high magnification of Fxr1p labelled myoblasts. In adult $X$. tropicalis, Fxr1p expression is confined to the cytoplasm of neurons in the brain (E) and all the spermatogenic cells in the testis (F). In addition, a granular labelling pattern is observed in skeletal muscle tissue (G) mal) and neural (ectodermal) tissue (Fig. 4B). From stage 37 onwards a further tissue-specific expression was observed with a high expression in the cytoplasm of neurons and skeletal muscle tissue (Fig. 4 C,D). In adult frogs, Fxr1p distribution remained tissue-specific. A strong labelling is observed in the cytoplasm of neurons (Fig. 4E), all the spermatogenic cells of the testis (Fig. 4F) and skeletal muscle tissue (Fig. 4G). This Fxr1p expression pattern during embryonic development and adult stages showed high similarities with the expression patterns described for man, mice and fish (Dube et al., 2000, Engels et al., 2004, Khandjian et al., 1998, Tamanini et al., 1997).

Both Fmrp and Fxr1p share the nuclear labelling during very early development which suggests a specific nuclear function during this short period of development. Most likely, translation of maternally provided transcripts account for these gene products. The high expression of Fxr1p in skeletal muscle tissue at late embryonic stages and in adult frogs suggests a role for Fxr1p in myogenesis. Similar to striated muscle tissue from mouse and zebrafish, the characteristic granular labelling of Fxr1p present in skeletal muscle from frogs suggests that Fxr1p is located in specific structures of the muscle tissue, called costameres (Dube et al., 2000, Engels et al., 2004, Mientjes et al., 2004). In man and mice, different isoforms of Fxr1p have been described for the different tissues, due to alternative splicing (Bakker et al., 2000, Huot et al., 2001, Khandjian et al., 1998, Tamanini et al., 1997). In contrast, Fmrp isoforms were identical for all the different tissues tested, that is, the presence of four major isoforms. Here, Western blotting was performed to study the presence of specific isoforms in frog tissues. Using antibody $\mathrm{KI}$, against Fmrp, only one major isoform of 72 $\mathrm{kDa}$ could be detected in both brain and testis tissue from $X$. tropicalis. As a control, homogenates from HEK293T cells trans- 
A

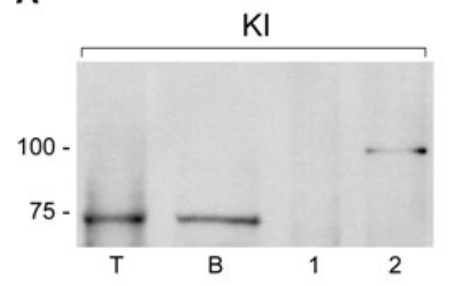

B

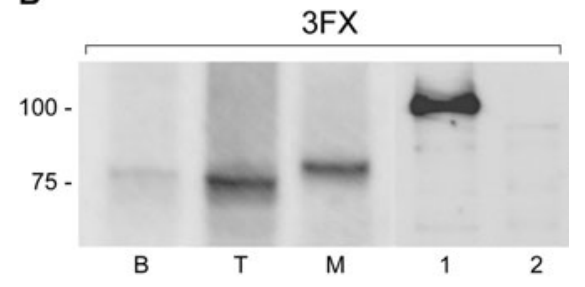

Fig. 5. Western blot analysis of adult $X$. tropicalis tissues using antibodies $\mathrm{KI}$ (A) and $3 \mathbf{F X}$ (B). (A) In homogenates from testis (lane T) and brain (lane B), a single isoform of approximately $72 \mathrm{kDa}$ could be detected. Importantly, homogenates of HEK293T cells transfected with XtFmr1EGFP showed a prominent band representing the Fmrp-EGFP fusion protein of approximately $99 \mathrm{kDa}$ (lane 2), whereas transfection with XtFxr1EGFP did not results in any detectable isoform (lane 1). (B) For Fxr1p, a weak band of approximately $80 \mathrm{kDa}$ could be detected in brain (lane B) and in testis this band was more prominent (lane T). In skeletal muscle homogenates a single isoform of approximately $84 \mathrm{kDa}$ appeared (lane M). The specificity of antibody $3 F X$ is illustrated by the detection of the FX1pEGFP fusionprotein in XtFxr1-EGFP transfected HEK293T cells with a size of approximately $107 \mathrm{kD}$ (lane 1) and the absence of any isoform in XtFmr1EGFP transfected HEK293T cells (lane 2).

fected with an expression construct containing EGFP-Fmr1 or EGFP-FXr1 were incubated with antibody KI. The lane loaded with the homogenate containing EGFP-Fmrp fusion proteins revealed a band of $99 \mathrm{kDa}$ (72kDa for Fmrp and $27 \mathrm{kDa}$ for EGFP), whereas the other lane loaded with EGFP-Fxr1p fusion proteins was totally devoid of specific isoforms (Fig. 5A). Antibody 3FX, against Fxr1p, showed the presence of a single molecular form of approximately $80 \mathrm{kDa}$ in homogenates from both brain and testis tissue. In skeletal muscle tissue Fxr1p is present as a prominent single isoform of approximately $84 \mathrm{kDa}$. The specificity of the 3FX antibody was illustrated by the presence and absence of cross reactive material in the lane loaded with a homogenate from HEK293T cells transfected with $X t F X r 1$ and $X t F m r 1$, respectively (Fig. 5B). These results demonstrate that $X$. tropicalis expresses fewer $F X r$ isoforms compared to man and mice.

In conclusion, we report here the characterization of Fmrp and Fxr1p in Xenopus tropicalis. Both proteins share the major functional domains with their murine and human orthologues and show a differential expression pattern. Importantly, the tissuespecific expression of both proteins in adult frogs is comparable to the other vertebrate systems; however, the number of isoforms is restricted to one major isoform, which makes this model more simple to study in vivo gene function. Additionally, Xenopus is a widely used developmental model organism with a fast external (transluscent) development, many eggs per breeding and the recent development of novel gene knockdown technology using the morpholino strategy. All together, this makes $X$. tropicalis an attractive complementary model system to study the physiological function of Fmrp and Fxr1p, especially during embryonic development.

\section{Experimental Procedures}

Xenopus tropicalis were obtained from the Hubrecht laboratory, Utrecht and originate from a 6th generation Nigeria inbred line. Methods of egg collection, fertilization and embryo culture were as described (Gao et al., 1994). Developmental stages were assigned according to Nieuwkoop and Faber (Nieuwkoop and Faber, 1994).

The Sanger Institute Xenopus tropicalis EST database was searched for orthologues (www.sanger.ac.uk/Projects/X. tropicalis/).

Sets of oligo nucleotide primers were designed and used to extract mRNA sequences by RT-PCR from either adult brain for XtFmr1 or whole embryos, stage 37. For XtFXr1 adult skeletal muscle was used.

Phylogenetic analysis has been performed using the Treetop phylogenetic tree prediction program from GeneBee Services (http:// www.genebee.msu.su)Rabbit antibody $\mathrm{KI}$ was raised against the Cterminus of FMRP (Adinolfi et al., 1999, Reis et al., 2004) while monoclonal 3FX antibody was raised against a synthetic polypeptide that was common to the long and short isoforms of FXR1P (Khandjian et al., 1998). HEK293T cells were transfected with lipofectamine PLUS according to the manufacturer. For immunofluorescence analysis of transfected cells either an anti-rabbit or anti-mouse secondary antibody conjugated with TRITC was used. The details of the Western blotting procedure and the indirect immuno-peroxidase method on paraffin sections has been described before (Bakker et al., 2000).

\section{Acknowledgements}

This work was supported by IOP Genomics (L.B.) and by NWO grant 908-02-010 (S.P.). Excellent photography of Tom de Vries Lentsch is greatly appreciated. We are grateful to Dr. B. Bardoni for providing antibody $3 F X$.

\section{References}

ADINOLFI, S., BAGNI, C., MUSCO, G., GIBSON, T., MAZZARELLA, L. and PASTORE, A. (1999). Dissecting fmr1, the protein responsible for fragile $\mathrm{x}$ syndrome, in its structural and functional domains. RNA 5: 1248-1258.

BAKKER, C.E., DE DIEGO OTERO, Y., BONTEKOE, C., RAGHOE, P., LUTEIJN, T., HOOGEVEEN, A.T., OOSTRA, B.A. and WILLEMSEN, R. (2000). Immunocytochemical and biochemical characterization of fmrp, fxr1p and fxr2p in the mouse. Exp Cell Res 258: 162-70.

BAKKER, C.E., VERHEIJ, C., WILLEMSEN, R., VANDERHELM, R., OERLEMANS, F., VERMEY, M., BYGRAVE, A., HOOGEVEEN, A.T., OOSTRA, B.A., REYNIERS, E. et al. (1994). Fmr1 knockout mice: A model to study fragile $x$ mental retardation. Cel/ 78: 23-33.

BARDONI, B. and MANDEL, J.L. (2002). Advances in understanding of fragile $x$ pathogenesis and fmrp function and in identification of $x$ linked mental retardation genes. Curr Opin Genet Dev 12: 284-93.

BONTEKOE, C.J., MCILWAIN, K.L., NIEUWENHUIZEN, I.M., YUVA-PAYLOR, L.A., NELLIS, A., WILLEMSEN, R., FANG, Z., KIRKPATRICK, L., BAKKER, C.E., MCANINCH, R. et al. (2002). Knockout mouse model for fxr2: A model for mental retardation. Hum Mol Genet 11: 487-98.

DUBE, M., HUOT, M.E. and KHANDJIAN, E.W. (2000). Muscle specific fragile $x$ related protein 1 isoforms are sequestered in the nucleus of undifferentiated myoblast. BMC Genet 1: 1-4.

ENGELS, B., VAN 'T PADJE, S., BLONDEN, L., SEVERIJNEN, L.A., OOSTRA, B.A. and WILLEMSEN, R. (2004). Characterization of fxr1 in danio rerio; a simple vertebrate model to study costamere development. J Exp Biol 207: 3329-38.

GAO, X., KUIKEN, G.A., BAARENDS, W.M., KOSTER, J.G. and DESTREE, O.H. 
(1994). Characterization of a functional promoter for the xenopus wnt-1 gene on vivo. Oncogene 9: 573-81.

HUOT, M.E., MAZROUI, R., LECLERC, P. and KHANDJIAN, E.W. (2001). Developmental expression of the fragile $x$-related 1 proteins in mouse testis: Association with microtubule elements. Hum Mol Genet 10: 2803-2811.

KHANDJIAN, E.W., BARDONI, B., CORBIN, F., SITTLER, A., GIROUX, S., HEITZ, D., TREMBLAY, S., PINSET, C., MONTARRAS, D., ROUSSEAU, F. et al. (1998). Novel isoforms of the fragile $x$ related protein fxr1p are expressed during myogenesis. Hum Mol Genet 7: 2121-2128.

MIENTJES, E.J., WILLEMSEN, R., KIRKPATRICK, L.L., NIEUWENHUIZEN, I.M., HOOGEVEEN-WESTERVELD, M., VERWEIJ, M., REIS, S., BARDONI, B., HOOGEVEEN, A.T., OOSTRA, B.A. et al. (2004). Fxr1 knockout mice show a striated muscle phenotype: Implications for fxr1p function in vivo. Hum Mol Genet 13: 1291-1302.

NIEUWKOOP, P.D. and FABER, J. (1994). Normal table of xenopus laevis (daudin). Garland Publishing, Inc., New York and London.
REIS, S.A., WILLEMSEN, R., VAN UNEN, L., HOOGEVEEN, A.T. and OOSTRA, B.A. (2004). Prospects of tat-mediated protein therapy for fragile $x$ syndrome. $\checkmark$ Mol Histol 35: 389-95.

TAMANINI, F., WILLEMSEN, R., VAN UNEN, L., BONTEKOE, C., GALJAARD, H., OOSTRA, B.A. and HOOGEVEEN, A.T. (1997). Differential expression of fmr1, fxr1 and fxr2 proteins in human brain and testis. Hum Mol Genet 6: 1315-1322.

WILLEMSEN, R., OOSTRA, B.A., BASSELL, G.J. and DICTENBERG, J. (2004). The fragile $x$ syndrome: From molecular genetics to neurobiology. Ment Retard Dev Disabil Res Rev 10: 60-7.

Received: January 2005 Reviewed by Referees: February 2005 Modified by Authors and Accepted for Publication: February 2005 